\section{Regulation of neuroblast competence: multiple temporal identity factors specify distinct neuronal fates within a single early competence window}

\author{
Michael D. Cleary and Chris Q. Doe ${ }^{1}$ \\ University of Oregon Department of Neuroscience, Howard \\ Hughes Medical Institute, Eugene, Oregon 97403, USA
}

Cellular competence is an essential but poorly understood aspect of development. Is competence a general property that affects multiple signaling pathways (e.g., chromatin state), or is competence specific for each signaling pathway (e.g., availability of cofactors)? Here we find that Drosophila neuroblast 7-1 (NB7-1) has a single early window of competence to respond to four different temporal identity genes (Hunchback, Krüppel, Pdm, and Castor); that each of these factors specifies distinct motor neuron identities within this competence window but not outside it; and that progressive restriction to respond to Hunchback and Krüppel occurs within this window. Our work raises the possibility that multiple competence windows may allow the same factors to generate different cell types within the same lineage.

Supplemental material is available at http://www.genesdev.org.

Received October 6, 2005; accepted in revised form December 21, 2005.

Cellular diversity is often achieved through the generation of multiple cell types from a single progenitor. One of the most striking examples of this process occurs in the CNS, where vast numbers of neurons and glia with unique functions are derived from a limited number of progenitors. Regulated expression of intrinsic and extrinsic cues that confer spatial and temporal identity is one mechanism for controlling the generation of cellular diversity in the CNS (Pearson and Doe 2004). However, a common feature of progenitor cells is that they do not remain competent to respond to such cues throughout development. Restriction of progenitor competence is an important mechanism ensuring that the correct progeny are made at the proper time and place.

The vertebrate CNS provides at least two examples of the role of progenitor competence in the generation of cellular diversity. The cerebral cortex is derived from progenitor cells whose progeny migrate to distinct layers depending on their time of birth (McConnell 1992).

[Keywords: Progenitor cell; competence; Krüppel; Hunchback; Pdm; Castor]

${ }^{1}$ Corresponding author.

E-MAIL cdoe@uoneuro.uoregon.edu; FAX (541) 346-4736.

Article and publication are at http://www.genesdev.org/cgi/doi/10.1101/ gad.1382206.
Clonal analysis and heterochronic transplant experiments have shown that early cortical progenitors are multipotent, but the ability to make early cell types is gradually lost as progenitors age, in a process known as "progressive restriction" (Desai and McConnell 2000). Both extrinsic and intrinsic cues appear to regulate cortical progenitor competence (Bohner et al. 1997; Hanashima et al. 2004). Restriction of competence also occurs during vertebrate retinal development, but instead of a progressive restriction of competence, retinal progenitors pass through distinct, nonoverlapping competency states (Cepko et al. 1996). While retinal progenitors at each stage can produce multiple cell types, once a competency state has been passed it is not possible to induce production of the earlier cell types. As with cortical progenitors, both extrinsic and intrinsic cues appear to regulate retinal progenitor competence /Waid and McLoon 1998; Belliveau and Cepko 1999; Rapaport et al. 2001).

While studies of cortical and retinal progenitors demonstrate the general significance of progenitor competence in generating cell diversity, little is known about the molecular basis of "competence" or "progressive restriction of competence". Is competence a general cellular state that allows changes in gene expression in response to multiple signaling pathways? Or is competence specific for each signaling pathway, perhaps reflecting the availability of a particular cofactor?

Here we investigate these questions using the wellcharacterized Drosophila neuroblast 7-1 (NB7-1) system (Fig. 1). NB7-1 is a neural progenitor that buds off a series of ganglion mother cells (GMC-1, GMC-2, GMC-3, etc; named by birth order), and each GMC makes two postmitotic neurons (U1/sib, U2/sib, U3/sib, etc; named by birth order); the U1-U5 neurons can be identified by their position and staining for Even-skipped (Eve) protein (Isshiki et al. 2001; Pearson and Doe 2003). Four different transcription factors-Hunchback $(\mathrm{Hb})$, Krüppel $(\mathrm{Kr})$, $\mathrm{Pdm} 1 / \mathrm{Pdm} 2$ (Pdm), and Castor (Cas)-are sequentially expressed in NB7-1 (Isshiki et al. 2001; Pearson and Doe 2003; Grosskortenhaus et al. 2005), similar to other neuroblast lineages (Kambadur et al. 1998; Brody and Odenwald 2000; Isshiki et al. 2001; Novotny et al. 2001; Kanai et al. 2005). NB7-1 has high $\mathrm{Hb}$ and low $\mathrm{Kr}$ during the first division (and maintained in the $\mathrm{U} 1$ neuron); low $\mathrm{Hb}$ and low $\mathrm{Kr}$ during the second division (and maintained in the U2 neuron); $\mathrm{Kr}$ alone is present during the third division (and maintained in the U3 neuron); Pdm alone is present during the fourth division (and transiently detected in the U4 neuron); and both Pdm and Cas are present during the fifth division (with Cas maintained in the U5 neuron) (Fig. 1; Isshiki et al. 2001). All four transcription factors specify temporal identity within the NB7-1 lineage. $\mathrm{Hb}$ is necessary and sufficient to specify $\mathrm{U} 1$ and $\mathrm{U} 2$ fates (high levels give U1, lower levels give U2) (Isshiki et al. 2001; Pearson and Doe 2003; Grosskortenhaus et al. 2005); $\mathrm{Kr}$ is necessary and sufficient to specify the U3 fate (Isshiki et al. 2001; this work); and Pdm specifies U4 cell fate, whereas Pdm/Cas together specify U5 cell fate (R. Grosskortenhaus and C.Q. Doe, unpubl.).

Most importantly for this work, the competence of NB7-1 to respond to $\mathrm{Hb}$ has been well-characterized 


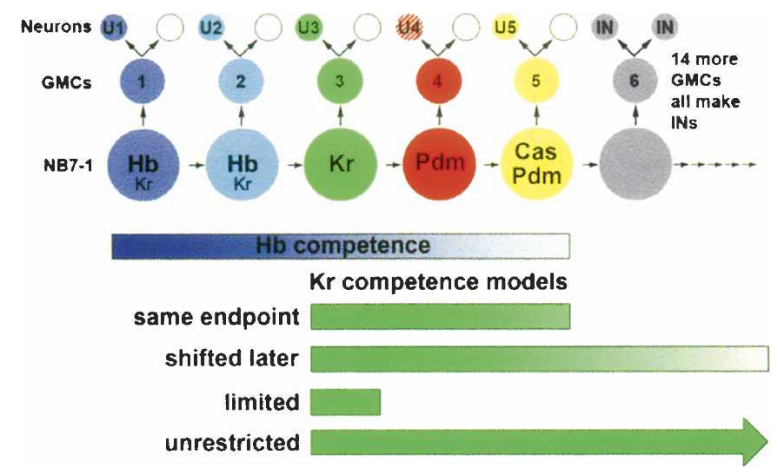

Figure 1. Temporal identity in the NB7-1 lineage. In the NB7-1 lineage, the first five GMCs give rise to the U1-U5 neurons and their siblings; and the following 15 GMCs produce $\sim 30$ interneurons (Schmid et al. 1999; Isshiki et al. 2001; Pearson and Doe 2003). Hb, $\mathrm{Kr}, \mathrm{Pdm}$, and Cas are sequentially expressed in the neuroblast and maintained in their neuronal progeny (except Pdm, which is transient in U4). Only GMC1-GMC5 and U1-U5 neurons are Eve ${ }^{+}$.

(Pearson and Doe 2003; Grosskortenhaus et al. 2005), so we can ask whether later temporal identity factors function within the same competence window. Pulses of $\mathrm{Hb}$ at different times in the lineage show that NB7-1 undergoes progressive restriction in competence to generate $\mathrm{U} 1 / \mathrm{U} 2$ neurons over time, and that the competence window ends after five cell divisions (Pearson and Doe 2003). If $\mathrm{Hb}$ levels are kept high throughout the lineage, however, the neuroblast will extend its competence window correspondingly, making many $\mathrm{U} 1 / \mathrm{U} 2$ neurons before resuming its normal lineage following $\mathrm{Hb}$ down-regulation (Grosskortenhaus et al. 2005). Thus, NB7-1 has a well-defined competence window for responding to $\mathrm{Hb}$ and extended expression of $\mathrm{Hb}$ can both extend this window and maintain the full competence of NB7-1 to produce later U neurons. Here we test whether the same competence window and maintenance of competence applies to multiple temporal identity factors. An identical competence window would support a general competence mechanism, whereas a different competence window for each temporal identity factor would suggest factor-specific competence mechanisms.

\section{Results and Discussion}

NB7-1 has a single competence window for responding to Hunchback and Krüppel

To determine whether NB7-1 undergoes progressive restriction in competence to respond to $\mathrm{Kr}$, similar to that observed for $\mathrm{Hb}$ (Pearson and Doe 2003), we generated pulses of $\mathrm{Kr}$ at progressively later points in the NB7-1 lineage. We used both $h s p 70-K r$ and $h s p 70-h b$ to allow us to precisely compare the effects of both genes (Fig. 2; a comparison of all $\mathrm{Hb}$ and $\mathrm{Kr}$ experiments are summarized in Supplementary Fig. 1). We confirmed that progressively later pulses of $\mathrm{Hb}$ produce a decreasing frequency of U1/U2 neurons (Fig. 2; Pearson and Doe 2003). Similarly, progressively later $\mathrm{Kr}$ pulses generated decreasing frequencies of extra $\mathrm{U} 3$ at each subsequent stage (Fig. 2), with the exception of the earliest portion of the lineage, where $\mathrm{Hb}$ is known to be dominant to $\mathrm{Kr}$ (Isshiki et al. 2001). Thus, NB7-1 shows progressive restriction in competence to respond to both $\mathrm{Hb}$ and $\mathrm{Kr}$, and compe- tence to respond to both $\mathrm{Hb}$ and $\mathrm{Kr}$ is lost at the same point in the lineage (after five divisions).

We next used an independent method to measure the competence window in the NB7-1 lineage. We used prospero-gal4 to induce expression of $\mathrm{Kr}$ within the NB7-1 lineage from the fourth division onward (Fig. 3A). When we use one copy of $U A S-K r$ at $22^{\circ} \mathrm{C}$, which provides relatively low levels of $\mathrm{Kr}$, we observed only five to six Eve ${ }^{+}$ U neurons (Fig. 3C), mostly U1, U2, and three U3 neurons $(91 \%)$, but also $U 1, U 2$, and four U3 neurons $(9 \%)$. Thus, NB7-1 loses competence to respond to prolonged $\mathrm{Kr}$ expression after five to six cell divisions, similar to results from the $\mathrm{Kr}$ pulse experiments described above. Prolonged expression of $\mathrm{Hb}$ using the same conditions (prospero-gal4, one copy of $U A S-H b, 22^{\circ} \mathrm{C}$ ) also results in just five to six Eve ${ }^{+}$U neurons (Pearson and Doe 2003). We conclude that NB7-1 has a single competence window for generating $\mathrm{U} 1-\mathrm{U} 3$ neurons in response to $\mathrm{Hb}$ and $\mathrm{Kr}$.

NB7-1 has a single competence window for responding to Krüppel, Pdm, and Castor

We next wanted to test whether the later-expressed temporal identity factors Pdm and Cas share the same early competence window with $\mathrm{Kr}$, or if they have distinct competence windows. Pdm specifies the U4 neuronal identity, while Pdm/Cas together specify U5 neuronal identity (R. Grosskortenhaus and C.Q. Doe, unpubl.). We used scabrous-gal4 to prolong $\mathrm{Kr}$ expression for a variable length of time within the NB7-1 lineage (two copies of $U A S-K r$ at $29^{\circ} \mathrm{C}$ ), which delayed but did not prevent the sequential expression of $\mathrm{Kr}, \mathrm{Pdm}$, and Cas (Fig. 4). This experiment allowed us to assay NB7-1 competence when presented with $\mathrm{Kr}, \mathrm{Pdm}$, or Cas at different times in its lineage.

We found that the scabrous-gal4 UAS-Kr embryos always had a total number of seven to eight Eve ${ }^{+} \mathrm{U}$ neurons, although ectopic U3 neurons ranged from two to six in number (Fig. 5). Interestingly, hemisegments with only two ectopic U3 neurons typically had U4/U5 neu-

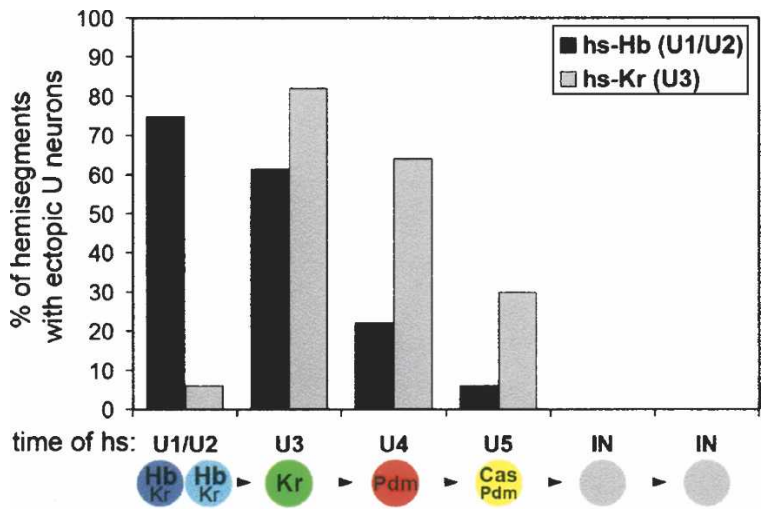

Figure 2. NB7-1 undergoes progressive restriction to respond to $\mathrm{Hb}$ and $\mathrm{Kr}$. Percentage of hemisegments with one or two extra Eve ${ }^{+}$" $U$ " neurons produced in response to heat-shock induced pulses of $\mathrm{Hb}$ (black, ectopic U1/U2 neurons) or Kr (gray, ectopic U3 neurons). Heat shock was applied just prior to the birth of each cell type listed in the lineage diagram below. (IN) Interneuron. The numbers of hemisegments scored in the $\mathrm{Hb}$ experiments are 44 (U1/U2), 57 (U3), 63 (U4), 66 (U5), and 50 (INs). The numbers for the Kr experiments are 50 (U1/U2), 50 (U3), 47 (U4), 66 (U5), and 100 (INs). 


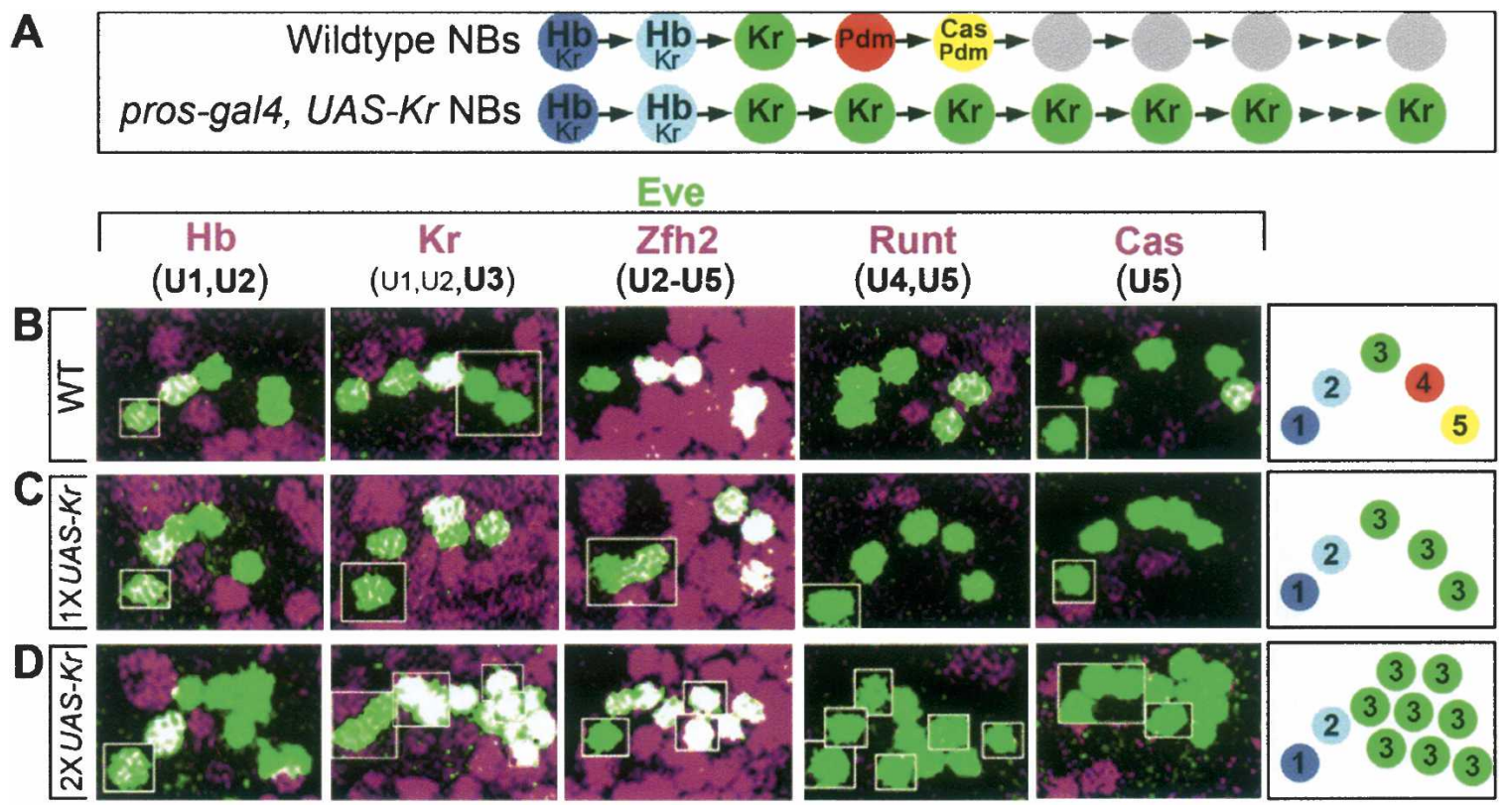

Figure 3. Prolonged Kr expression can extend NB7-1 competence. (A) Schematic of wild-type and experimental temporal identity factor profile within the NB7-1 lineage. $(B-D)$ One hemisegment of a stage 17 embryo is shown stained for the indicated markers; a summary of $U$ neuron phenotype is shown in the box to the right. Anterior up, midline to left. $(B)$ Wild type. $(C)$ Low $\mathrm{Kr}$ misexpression $\left(1 \times U A S-K r, 22^{\circ} C_{;} n=66\right.$ hemisegments). (D) High $\mathrm{Kr}$ misexpression ( $2 \times U A S-K r, 29^{\circ} \mathrm{C} ; \mathrm{n}=70$ hemisegments).

rons; those with three ectopic U3 neurons had only a U4 neuron, and those with four or more ectopic U3 neurons lacked both U4/U5 neuronal fates (Fig. 5B,C). We interpret these data in the following way. In segments where $\mathrm{Kr}$ declines the fastest (fewest ectopic U3 neurons), there is time for Pdm to induce U4 fate and Pdm/Cas to induce U5 fates prior to loss of competence; however, in segments where $\mathrm{Kr}$ lasts the longest, both Pdm and Cas expression occur after the competence window and no U4/U5 fates are produced. Taken together, this experiment allows us to draw several conclusions (summarized in Fig. 5D): (1) Prolonged Kr expression can partially extend the neuroblast competence window (from five to six divisions to seven to eight divisions); (2) competence to respond to $\mathrm{Kr}, \mathrm{Pdm}$, and Cas is simultaneously lost at the end of this competence window, suggesting that there is a single competence window for responding to multiple temporal identity factors; and (3) each temporal identity factor specifies different U1-U5 motor neuron identities within the competence window, but not outside it. It is currently an open question as to how prolonged expression of one factor $\left(\mathrm{Kr}_{\text {; }}\right.$ this work; $\mathrm{Hb}$, Grosskortenhaus et al. 2005) can extend the competence window to respond to three distinct factors $(\mathrm{Kr}, \mathrm{Pdm}$, and Cas).

Loss of neuroblast competence requires down-regulation of Hunchback but not Krüppel

Our previous experiment showed that prolonging $\mathrm{Kr}$ expression (scabrous-gal4 UAS-Kr) in NB7-1 lineage can only partially extend neuroblast competence. Interestingly, similar experiments prolonging $\mathrm{Hb}$ expression (scabrous-gal4 UAS-hb) revealed that the neuroblast maintains full competence for as long as $\mathrm{Hb}$ is expressed, in some cases over 15 divisions, with normal U3-U5 fates appearing after $\mathrm{Hb}$ levels decline (Grosskortenhaus et. al. 2005). Thus, extended $\mathrm{Hb}$ expression-but not extended $\mathrm{Kr}$ expression-can maintain the neuroblast in a young, fully competent state. This raised the possibility that down-regulation of $\mathrm{Hb}$ is required for loss of neuroblast competence; alternatively, $\mathrm{Hb}$ may be more potent than $\mathrm{Kr}$ in maintaining neuroblast competency.

To distinguish these models, we compared the effect of high-level $\mathrm{Hb}$ or $\mathrm{Kr}$ expression beginning at the fourth neuroblast division (prospero-gal4, $2 \times U A S-h b$ or UAS$K r, 29^{\circ} \mathrm{C}$ ), which would allow $\mathrm{Hb}$ down-regulation and permit us to compare the efficacy of $\mathrm{Hb}$ versus $\mathrm{Kr}$ in extending neuroblast competence. Performing this experiment with $\mathrm{Hb}$ resulted in a partial extension of neuroblast competence and the production of an average of 9.1 Eve $^{+}$U neurons: U1-U3, 6.1 extra U1, and no U4/U5 (Pearson and Doe 2003). Performing the experiment with $\mathrm{Kr}$ resulted in an almost identical phenotype of $9.8 \mathrm{Eve}^{+}$ U neurons: U1/U2, $7.8 \mathrm{U} 3 \mathrm{~s}$, and no U4/U5 (Fig. 3D). Thus, $\mathrm{Hb}$ and $\mathrm{Kr}$ appear equally efficient at extending neuroblast competence; this is supported by their equivalent effect when expressed under heat shock or lower level prospero-gal4 control (competence lost after five divisions) (Figs. 2, 3C; Pearson and Doe 2003). More importantly, a comparison of the scabrous-gal4 UAS-hb and prospero-gal4 UAS-hb experiments shows that $\mathrm{Hb}$ down-regulation is critical for loss of neuroblast competence. When $\mathrm{Hb}$ is maintained from the beginning of the lineage (scabrous-gal4 UAS-hb), competence persists for the length of $\mathrm{Hb}$ expression, in some cases over 15 divisions; when $\mathrm{Hb}$ down-regulation occurs followed by permanent $\mathrm{Hb}$ re-expression one division later (prosperogal4 UAS-hbl, then competence is lost after approximately nine divisions (Pearson and Doe 2003; Grosskortenhaus et al. 2005). We conclude that downregulation of $\mathrm{Hb}$, but not $\mathrm{Kr}$, initiates progressive restriction in neuroblast competence that is normally complete after five divisions. 


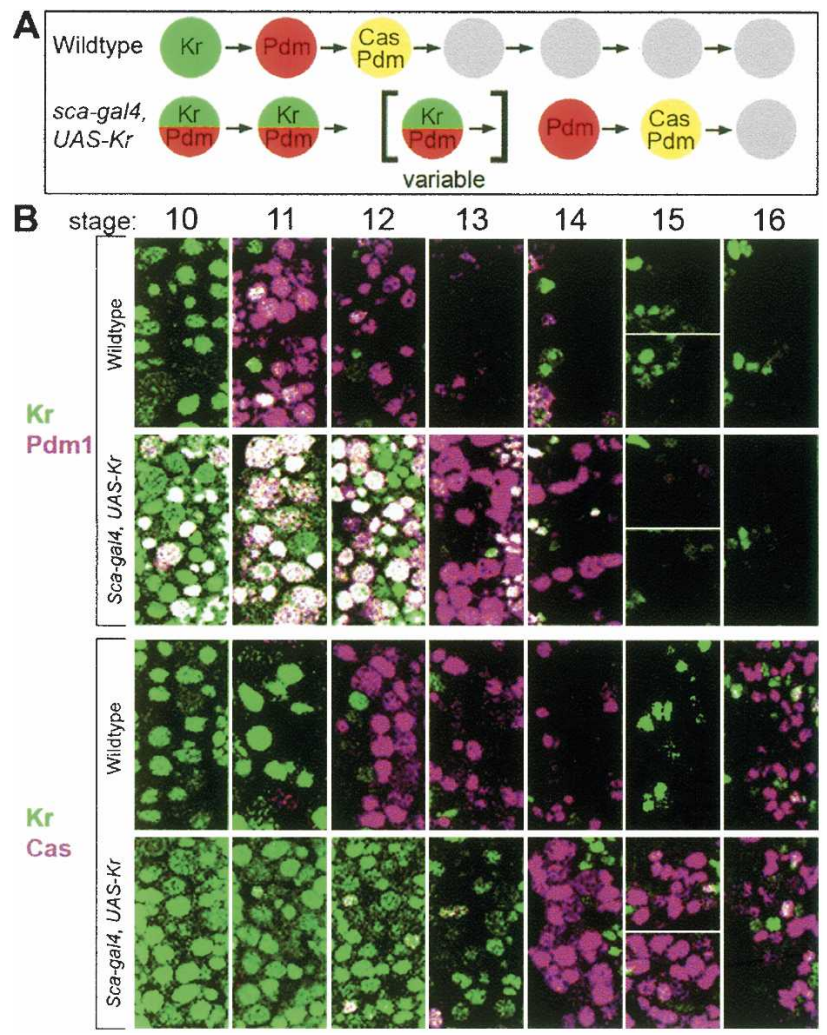

Figure 4. Prolonged Kr expression delays but does not prevent sequential Pdm and Cas expression. (A) Schematic of wild-type and experimental temporal identity factor profile within the entire neuroblast layer of wild-type and experimental embryos. $(B)$ Single confocal image of neuroblasts within two hemisegments from embryonic stage 10-16. In wild-type neuroblasts, $\mathrm{Kr}$ expression peaks at stage 10, Pdm expression peaks at stage 11, and Cas expression peaks at stage 12 (Grosskortenhaus et al. 2005). In scabrous-gal4 $U A S-K r$ neuroblasts, $\mathrm{Kr}$ expression persists for variable lengths of time (on average peaking at stage 12), Pdm persists through stage 13 (it also shows early activation due to precocious $\mathrm{Kr}$ expression, as previously described; Isshiki et. al. 2001), and Cas persists through stage 14 and beyond. We observed no sign of neuroblast cell cycle arrest, neuroblast cell death, or cell death of $\mathrm{Pdm}^{+}$or $\mathrm{Cas}^{+}$neurons (Supplementary Fig. 5; data not shown).

Mitotic progenitors, but not post-mitotic neurons, are competent to respond to Krüppel

Thus far, we have been investigating how neuroblast competence changes over multiple rounds of cell division. Here, we consider how competence changes during neuronal differentiation. We expressed $\mathrm{Kr}$ in high levels in the newborn post-mitotic U1-U5 neurons leve-gal4 $U A S-K r)$. In these embryos, $\mathrm{Kr}$ is first detected just as the U1-U5 neurons are born. Despite high levels of $\mathrm{Kr}$ protein, no change in U1-U5 fate was ever detected (Supplementary Fig. 2). Conversely, transient expression of $\mathrm{Kr}$ in NB7-1/GMCs can occasionally generate ectopic U3 neurons that do not maintain $\mathrm{Kr}$ expression (Supplementary Fig. 3), despite the ability of $\mathrm{Kr}$ to positively autoregulate within the CNS (Supplementary Fig. 4). Thus, mitotic progenitors but not post-mitotic neurons are competent to respond to $\mathrm{Kr}$. Similar results have been observed for competence to respond to $\mathrm{Hb}$ (Pearson and Doe 2003).

\section{Conclusions and future directions}

Our experiments, combined with previous studies (Isshiki et al. 2001; Pearson and Doe 2003; Grosskortenhaus et al. 2005), allow us to make four major conclusions.

(1) A single early competence window is used by multiple temporal identity factors. The molecular basis for the early competence window is unknown, but it must be general enough to modulate response to four distinct transcription factors rather than being factor specific. Perhaps loss of competence leads to restricted access of $\mathrm{Hb}, \mathrm{Kr}, \mathrm{Pdm}$, and Cas to target loci involved in U1-U5 neuronal specification; other loci may remain unaffected, allowing these four transcription factors to induce different cell fates later in the neuroblast lineage (see below). Identifying $\mathrm{Hb}$ and $\mathrm{Kr}$ target genes, and in-

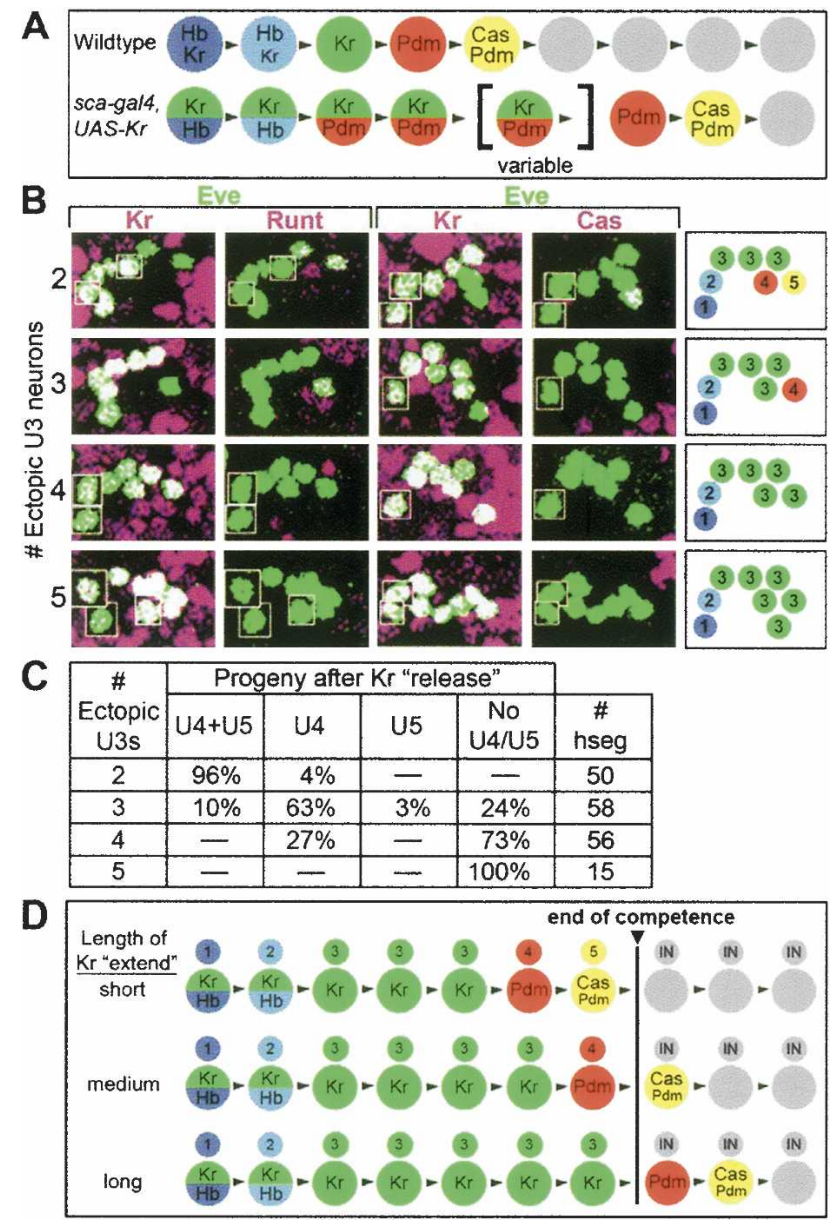

Figure 5. NB7-1 has a single competence window for responding to Krüppel, Pdm, and Castor. (A) Schematic of wild-type and experimental temporal identity factor profile within the entire neuroblast layer of wild-type and experimental embryos. $(B)$ One hemisegment of a stage 17 embryo is shown stained for the indicated markers; a summary of $\mathrm{U}$ neuron phenotype is shown in the box to the right. Anterior up, midline to left. The number of ectopic U3 neurons produced during the extended $\mathrm{Kr}$ expression is listed on the left. $(C)$ Quantitation of U4/U5 neurons depending on the number of ectopic U3 neurons in the hemisegment. (D) Interpretation of results. Extended $\mathrm{Kr}$ expression results in a delay in Pdm and Cas expression; $\mathrm{Kr}, \mathrm{Pdm}$, or Cas expressed during the competence window can induce U3-U5 fates, but not after the end of the competence window (black line). 
vestigating how or whether they undergo chromatin modifications during the process of progressive restriction will help resolve this question, and may provide insight into the mechanism of progressive restriction in mammalian neural progenitors.

(2) Each temporal identity factor specifies distinct motor neuron fates within the competence window, but not outside of it. Within the early competence window, each temporal identity factor specifies a unique U1-U5 neuronal identity: high $\mathrm{Hb}, \mathrm{U} 1$; low $\mathrm{Hb}, \mathrm{U} 2 ; \mathrm{Kr}, \mathrm{U} 3$; Pdm, U4; Pdm/Cas, U5. The loss of competence to generate U1-U5 fates may allow each of these transcription factors to be "reused" later in the NB7-1 lineage to generate different subsets of neurons. This model is supported by the fact that we and others ( $T$. Isshiki, pers. comm.) have observed a second round of $\mathrm{Kr}$ and Cas neuroblast expression later in embryonic development (Fig. 4). These findings suggest that neuroblasts have the potential for cycling through distinct competence windows, and may provide a model for understanding how successive competency states are established (e.g., in vertebrate retinal progenitors).

(3) NB7-1 undergoes progressive restriction in competence to respond to both $\mathrm{Hb}$ and $\mathrm{Kr}$. Competence to respond to both $\mathrm{Hb}$ and $\mathrm{Kr}$ is progressively restricted early in the lineage, then completely lost after five divisions of NB7-1. Progressive restriction may be regulated autonomously in the neuroblast or by changing environmental cues, such as inhibitory feedback from GMC or neuronal progeny. We favor a lineage-intrinsic mechanism because different neuroblasts lose competence to respond to $\mathrm{Hb}$ at different times (e.g., NB7-1 remains competent to respond to $\mathrm{Hb}$ for five divisions, whereas the adjacent NB1-1 is only competent to respond to $\mathrm{Hb}$ for two to three divisions) (Isshiki et al. 2001). A feedback inhibition model would have parallels with vertebrate retinal progenitors, where differentiated amacrine cells send an inhibitory feedback signal to terminate amacrine cell production (Belliveau and Cepko 1999). In this case, the signal would likely depend on the number of progeny produced rather than the type of progeny, because loss of competence can occur without production of the last-born neurons in the competence window (U4/ U5) (see Figs. 3, 5).

(4) Down-regulation of $\mathrm{Hb}$ but not $\mathrm{Kr}$ initiates progressive restriction and loss of competence. Neuroblast competence is maintained if $\mathrm{Hb}$ is expressed from the beginning of the lineage (Grosskortenhaus et al. 2005). However, neuroblast competence is not maintained when $\mathrm{Kr}$ is expressed from the beginning of the lineage (where $\mathrm{Hb}$ is down-regulated normally) (Fig. 5) or when $\mathrm{Hb}$ or $\mathrm{Kr}$ are expressed later in the lineage after normal $\mathrm{Hb}$ down-regulation (Figs. 2, 3; Pearson and Doe 2003). We propose that $\mathrm{Hb}$ down-regulation initiates progressive restriction in neuroblast competence, ultimately leading to a complete loss of competence.

\section{Materials and methods}

Fly stocks

(1) hsp70-Kr/CyO hb-lacZ; (2) hsp70-hb (Hb476.2)/CyO; hsp70-hb (HB476.1)/TM3; (3) yw; UAS-Kr; UAS-Kr; (4) yw; UAS-Kr; (5) prosperogal4 homozygous on chromosomes II and III; (6) eve-gal4[+3.5-4.3] homozygous on chromosome II; (7) scabrous-gal4 homozygous on chromosome II; (8) engrailed-gal4 homozygous on chromosome II.

Unless otherwise noted, all Gal4 lines were crossed to yw; UAS-Kr; $U A S-K r$ flies, and embryos were collected at $29^{\circ} \mathrm{C}$.
Heat-shock experiments

Embryos were collected for $1 \mathrm{~h}$, aged to the indicated time, and subjected to two cycles of $30 \mathrm{~min}$ at $37^{\circ} \mathrm{C}$, then $30 \mathrm{~min}$ at $22^{\circ} \mathrm{C}$, and allowed to develop to stage 16-17. This generates high levels of $\mathrm{Hb}$ and $\mathrm{Kr}$ without affecting control embryos. Before heat shock, some embryos were fixed and stained with Eve/Hb or Eve/Kr for developmental staging. When scoring the frequency of extra cells per hemisegment, no more than five hemisegments were scored per embryo (typically just one side of the CNS) so that, on average, at least 10 different embryos were used in the analysis of each timepoint.

\section{Protein immunolocalization}

Antibody staining was performed according to standard methods (Grosskortenhaus et al. 2005). Primary antibodies, dilutions, and sources are guinea pig anti-Hb, 1:400, East Asian Distribution Center for Segmentation Antibodies (EADC); guinea pig anti-Kr, 1:400, EADC; rabbit anti-Pdm1, 1:400, W. Chia (Temasek Lifesciences Laboratory and Department of Biological Sciences, National University of Singapore, Singapore); rabbit anti-Cas, 1:500, W. Odenwald (Neural Cell-Fate Determinants Section, National Institute of Neurological Disorders and Stroke, National Institutes of Health, Bethesda, MD); guinea pig anti-Eve, 1:400, EADC; mouse anti-Eve monoclonal 2B8, 1:20; guinea pig anti-Runt, 1: 400, EADC; rabbit anti-Runt, 1:500 of 1:10 preabsorbed, A. Brand (Wellcome Trust/Cancer Research UK Institute and Department of Genetics, University of Cambridge, Cambridge, UK); rat anti-Zfh2, 1:200, M. Lundell (Division of Life Sciences, University of Texas at San Antonio, San Antonio, TX); and sheep anti-Digoxigenin, 1:1000, Boehringer. Speciesspecific secondary antibodies were conjugated to Alexa 488, Alexa 555 (Molecular Probes), Cy5 (Jackson ImmunoResearch), or Biotin (Vector Labs) and were used at 1:200. The TSA-plus Fluorescein System (PerkinElmer) was used to detect the anti-Pdml antibody. All images were collected as confocal image stacks on a Leica TCS SP2 AOBS confocal microscope, processed in Image (NIH), and shown as two-dimensional projections. In all experiments, analysis was restricted almost exclusively to the abdominal segments of the CNS.

\section{Acknowledgments}

We thank Ruth Grosskortenhaus, Takako Isshiki, Bret Pearson, and Seth Blackshaw for commenting on the manuscript, and A. Brand, W. Chia, M. Lundell, and W. Odenwald for antibodies. This work was funded by NIH HD27056 (C.Q.D.); C.Q.D. is an HHMI Investigator.

\section{References}

Belliveau, M.J. and Cepko, C.L. 1999. Extrinsic and intrinsic factors control the genesis of amacrine and cone cells in the rat retina. Development 126: 555-566.

Bohner, A.P., Akers, R.M., and McConnell, S.K. 1997. Induction of deep layer cortical neurons in vitro. Development 124: 915-923.

Brody, T. and Odenwald, W.F. 2000. Programmed transformations in neuroblast gene expression during Drosophila CNS lineage development. Dev. Biol. 226: 34-44.

Cepko, C.L., Austin, C.P., Yang, X., Alexiades, M., and Ezzeddine, D. 1996. Cell fate determination in the vertebrate retina. Proc. Nat1. Acad. Sci. 93: 589-595.

Desai, A.R. and McConnell, S.K. 2000. Progressive restriction in fate potential by neural progenitors during cerebral cortical development. Development 127: 2863-2872.

Grosskortenhaus, R., Pearson, B.J., Marusich, A., and Doe, C.Q. 2005. Regulation of temporal identity transitions in Drosophila neuroblasts. Dev. Cell 8: 193-202.

Hanashima, C., Li, S.C., Shen, L., Lai, E., and Fishell, G. 2004. Foxg1 suppresses early cortical cell fate. Science 303: 56-59.

Isshiki, T., Pearson, B., Holbrook, S., and Doe, C.Q. 2001. Drosophila neuroblasts sequentially express transcription factors which specify the temporal identity of their neuronal progeny. Cell 106: 511-521.

Kambadur, R., Koizumi, K., Stivers, C., Nagle, J., Poole, S.J., and Odenwald, W.F. 1998. Regulation of POU genes by castor and hunchback establishes layered compartments in the Drosophila CNS. Genes \& Dev. 12: 246-260.

Kanai, M.I., Okabe, M., and Hiromi, Y. 2005. seven-up controls switching of transcription factors that specify temporal identities of Drosophila 


\section{Cleary and Doe}

neuroblasts. Dev. Cell 8: 203-213.

McConnell, S.K. 1992. The control of neuronal identity in the developing cerebral cortex. Curr. Opin. Neurobiol. 2: 23-27.

Novotny, T., Eiselt, R., and Urban, J. 2001. Hunchback is required for the specification of the early sublineage of neuroblast 7-3 in the Drosophila central nervous system. Development 129: 1027-1036.

Pearson, B.J. and Doe, C.Q. 2003. Regulation of neuroblast competence in Drosophila. Nature 425: 624-628.

- 2004. Specification of temporal identity in the developing nervous system. Annu. Rev. Cell. Dev. Biol. 20: 619-647.

Rapaport, D.H., Patheal, S.L., and Harris, W.A. 2001. Cellular competence plays a role in photoreceptor differentiation in the developing Xenopus retina. J. Neurobiol. 49: 129-141.

Schmid, A., Chiba, A., and Doe, C.Q. 1999. Clonal analysis of Drosophila embryonic neuroblasts: Neural cell types, axon projections and muscle targets. Development 126: 4653-4689.

Waid, D.K. and McLoon, S.C. 1998. Ganglion cells influence the fate of dividing retinal cells in culture. Development 125: 1059-1066. 


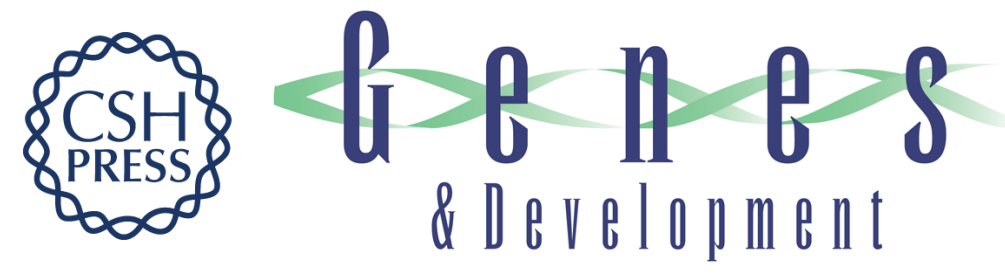

\section{Regulation of neuroblast competence: multiple temporal identity factors specify distinct neuronal fates within a single early competence window}

Michael D. Cleary and Chris Q. Doe

Genes Dev. 2006, 20:

Access the most recent version at doi:10.1101/gad.1382206

Supplemental http://genesdev.cshlp.org/content/suppl/2006/02/01/20.4.429.DC1

Material

References This article cites 17 articles, 8 of which can be accessed free at: http://genesdev.cshlp.org/content/20/4/429.full.html\#ref-list-1

License

Email Alerting Receive free email alerts when new articles cite this article - sign up in the box at the top Service right corner of the article or click here.

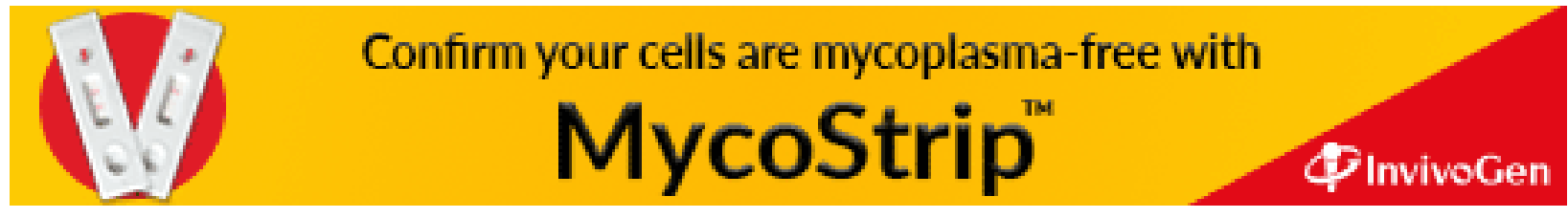

